\title{
PATTERN DENSITY BASED PREDICTION FOR DEEP REACTIVE ION ETCH (DRIE)
}

\author{
Tyrone F. Hill, Hongwei Sun, *Hayden K. Taylor, Martin A. Schmidt, and Duane S. Boning \\ Microsystems Technology Laboratories \\ Massachusetts Institute of Technology \\ Cambridge, MA 02139, USA \\ *Cambridge University \\ Engineering Department \\ Cambridge CB2 1PZ, UK
}

\begin{abstract}
A quantitative model capturing Deep Reactive Ion Etch (DRIE) pattern density effects is presented. Our previous work has explored the causes of wafer-level variation and demonstrated dielevel interactions resulting from pattern density and reactant species consumption [1]. Several reports have focused on experimental evidence and modeling of feature-level (aspect ratio) dependencies [2]. In contrast, in this work we contribute a computationally efficient and effective modeling approach that focuses on layout pattern density-induced nonuniformity in DRIE. This is a key component in an integrated model combining wafer-, die-, and feature-level DRIE dependencies to predict etch depth for an input layout and a characterized etch tool and process. A microscale engine turbopump layout is used to demonstrate the model, which was calibrated to fit across-die variation within $1 \%$ and intra-die variation to within $0.1 \%$ (normalized RMS error).
\end{abstract}

\section{INTRODUCTION}

Deep Reactive Ion Etch (DRIE) is a key technology for realizing the high aspect-ratio structures that are so prevalent in MEMS. The Bosch etch method used here consists of timemultiplexed cycles of polymer deposition $\left(\mathrm{C}_{4} \mathrm{~F}_{8}\right.$ feed gas $)$ and chemical etching of silicon ( $\mathrm{SF}_{6}$ feed gas). Recent work on DRIE has included optimizing process parameters [3], exploring aspect ratio dependencies [2], and identifying die-to-die effects (Sun et al.) [1]. Our work seeks to develop a model to capture and predict pattern or layout dependent effects in DRIE, as summarized in Fig. 1.

In this paper, we focus on the center component in Fig. 1, where we contribute a pattern density based model. By pattern density, we mean the area fraction of exposed surface on the wafer (that portion not blocked by an etch mask), and thus we are concerned with design or layout-specific variation in etch depth or rate due to pattern density variations across the chip and wafer. Our pattern density model convolves an etch "impulse response" with a local pattern density map for any given device layout, in order to predict die-level etch perturbations for that design. The layout for the full wafer (typically with multiple die) is analyzed, so that pattern-density based interactions between die are captured, as well as within-die pattern density perturbations. In addition, wafer level nonuniformities based on an empirically derived model are applied, resulting in predicted etch maps for each die across the wafer.

In the following, we first present the pattern density model. This is followed by discussion of feature-level effects, and a brief summary of the previously developed wafer-level variation model used in the framework of Fig. 1. Application of the model to a microscale turbopump is then presented. Finally, limitations in the current model and directions for future work are summarized.

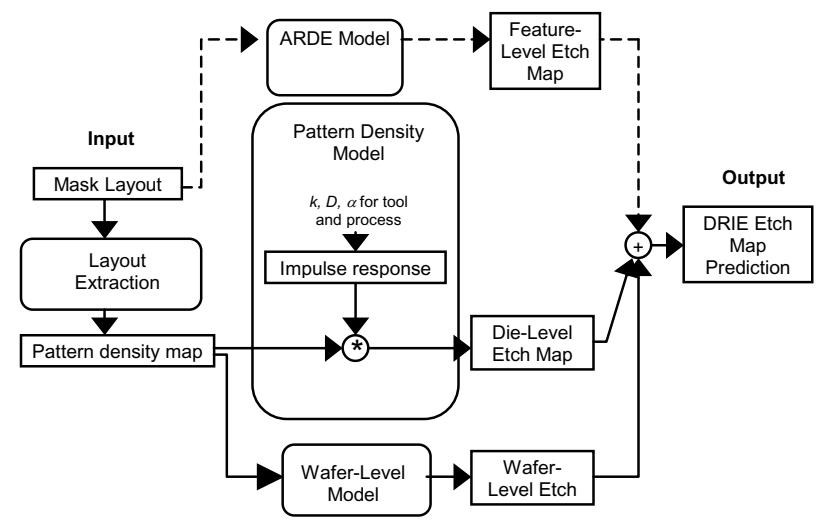

Figure 1. Framework for pattern dependent modeling in DRIE. The solid lines show components included in the present work.

\section{DIE-LEVEL MODEL}

The physical rationale behind the pattern density model is next described, followed by model derivation and implementation.

\section{Physical Motivation for Pattern Density Effects}

The Bosch process involves two major mechanisms: ionassisted dry chemical etching, and the deposition of a polymer inhibiting layer. During the etch cycle, we assume that the ionassisted etch rate will be affected by the concentration of the reactant species. That is to say, we assume key reactions are:

$$
\begin{aligned}
& S F_{6}+e^{-} \longrightarrow S_{x} F_{y}+S_{x} F_{y}{ }^{-}+F^{\bullet}+e^{-} \\
& S i+n F^{\bullet} \longrightarrow S i F_{n}
\end{aligned}
$$

Eq. 1 represents the dissociation of $\mathrm{SF}_{6}$ into neutral fluorine radicals and ions. The fluorine radicals reach the wafer by diffusion, and are responsible for removing silicon. We assume that localized concentration gradients of fluorine radicals create spatial variations in etch rate. Pattern density dependencies thus result from the localized consumption of reactants on different regions of the wafer. We find that these depressions in concentration can occur on relatively long length scales, i.e., across several millimeters, and thus result in interaction between multiple die on a wafer. Qualitatively, die that are surrounded by highly loaded areas will etch more slowly, since they encounter more competition for reactants.

\section{Pattern Density Model Development}

Die-level interactions are empirically modeled through the use of an "etch impulse response." This idea is analogous to a filterbased inter-layer dielectric (ILD) thickness prediction scheme for CMP proposed by Ouma et al. [4]. If $f(x, y)$ represents the spatial response to an impulse of pattern density, and $d(x, y)$ is a function 
describing the local spatial pattern density of a layout, then the die-level variation $z(x, y)$ is given by a convolution operation:

$$
z(x, y)=f(x, y) \otimes d(x, y)
$$

In our current model, the impulse response is based on the diffusion equation solution for a spherical coordinate system with an inverse distance $(1 / r)$ dependence. An expression for the filter function is obtained for the reduction $C$ of reactant concentration at a radius $r$ from an arbitrary point on the wafer surface. Eq. 7 represents diffusion of the species $C$, with surface area limited to a half sphere. By rearranging terms and integrating we can obtain an expression for the concentration (Eq. 8). In this equation, there are two important parameters: the reaction rate $k$ and the diffusion coefficient $D$, which represent the consumption rate of silicon and transport rate of etchant to the wafer surface.

$$
\begin{aligned}
& 2 \pi r^{2} D \frac{\partial C}{\partial r}=k \\
& C=-\frac{k}{D} \frac{1}{2 \pi r}
\end{aligned}
$$

Based on Eq. 8, we have the (negative) impact on background reactant concentration as a function of distance away from each area of exposed silicon. This impulse response thus forms a filter, as given by Eq. 9 below and illustrated in Fig. 2, that we can convolve with a representation of the open area (local pattern density) across the wafer. We allow for an empirical constant $\alpha$ in order to scale the filter with respect to wafer-level effects.

$$
f=-\alpha\left(\frac{k}{D}\right) \frac{1}{2 \pi r}
$$

While Eq. 9 gives a $1 / r$ spatial dependence and a magnitude scaling parameter (the aggregate of $k, D, \alpha$, and constants), other spatial forms may also be appropriate. For example, solution of the diffusion equation in cylindrical coordinates gives rise to a $\ln (r)$ dependence. An alternative approach is to empirically fit the spatial dependence, e.g., to $a /(c+r)^{b}$, allowing constants $a, b$, and $c$ to be determined empirically.

\section{Pattern Density Model Implementation}

In our implementation of the etch variation model (as summarized in Fig. 1), the pattern density model consists of the information related to the filter function. Specifically, the pattern density model consists of the filter function structure $(1 / r$ in this case), and an aggregate scaling parameter which is fit using characterization data for a fixed etch recipe. An AutoCAD layout for the design of interest is processed through a layout density extraction tool (provided courtesy of Praesagus, Inc.). The local layout pattern density map is convolved with the filter function to produce the pattern density perturbation to the etch rate or depth. Increased computational efficiency may be achieved by using an FFT approach rather than direct convolution. We assume that wafer-, die, and feature-level etch perturbations are additive: pattern density based variation is added to a wafer-level uniformity prediction to produce an etch variation map for the entire wafer

A set of test masks was designed to fit the pattern density model for a specific recipe. The masks have a small region (or a pair of regions) on the wafer consisting of concentric circles with pattern densities varying from 10 to $90 \%$. Etch experiments are performed and etch depths measured at a variety of distances from the patterned regions. These give the spatial extent and magnitude of pattern density-induced etch perturbation to fit the model. The filter parameters were extracted on a $1 \mathrm{~mm}$ x $1 \mathrm{~mm}$ size grid; additional amplitude scaling of the filter may be necessary when applied to a layout having a smaller discretization.

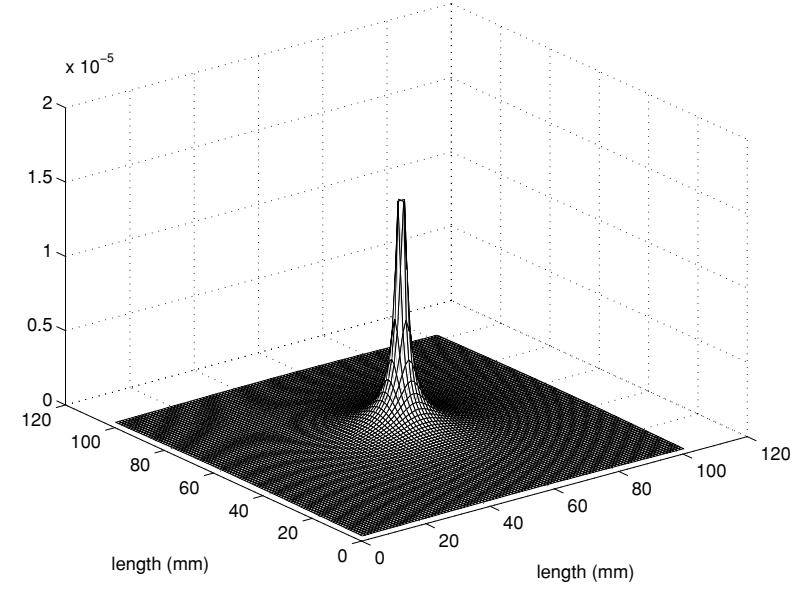

Figure 2. 3-d image of filter impulse response $f(x, y)$

For the experiments shown here, the recipe had a pressure trip of $94.0 \mathrm{mT}$ (meaning that the upper pressure limit for the chamber is $94 \mathrm{mT}$ ) and an automated pressure control value (APC) setting of $75^{\circ}$. The etch cycle used a $105 \mathrm{sccm}$ flow rate $\left(\mathrm{SF}_{6}\right), 100 \mathrm{~W}$ of platen power, and $750 \mathrm{~W}$ coil power. The passivation cycle had a $40 \mathrm{sccm}$ flow rate $\left(\mathrm{C}_{4} \mathrm{~F}_{8}\right), 60 \mathrm{~W}$ of platen power, and $600 \mathrm{~W}$ of coil power. The experiments were carried out in an Inductively Coupled Plasma (ICP) etcher manufactured by Surface Technology Systems of Newport, UK.

\section{FEATURE-LEVEL MODEL}

Feature-level effects include variations due to differing aspect ratios (depth to width) in individual etch features. It is thought that these variations are related to the probability of reactant transmission to the bottom of the feature, as described by Coburn and Winters [5]. Recent efforts by Yeom et al. to separate effects suggest a connection between pattern density and feature level uniformity [2]. Our work does not presently include feature-level effects; addition of an existing or new feature-scale model must be done with care to be consistent with the pattern density model.

\section{WAFER-LEVEL MODEL}

Wafer-level variation spans the entire wafer, and is related to ion and neutral transport in the plasma, as well as asymmetries in the geometry of the chamber [1]. For low loading densities ion transport is believed to be the dominant factor in determining uniformity. As a result, layouts with low loading $(<10 \%)$ generally exhibit a "hot spot" with a higher than average etch rate. As the etch rate increases, the behavior is governed by neutral transport, resulting in a "cold spot."

For the etch variation model in this work, we use an empirical characterization of the wafer-level variation for a given recipe. A separate set of test masks with uniformly spaced measurement locations is used. Each mask has a different uniform pattern density, and thus the wafer-level uniformity map corresponding to different global loadings can be empirically captured.

Fig. 3 shows a normalized (to highest etch rate location) wafer-level spatial etch map corresponding to $4.4 \%$ global loading. In some etch applications (including the turbopump example shown later), the wafer is rotated multiple times during the total etch. The wafer level model thus averages the spatial uniformity map through equivalent rotations. 


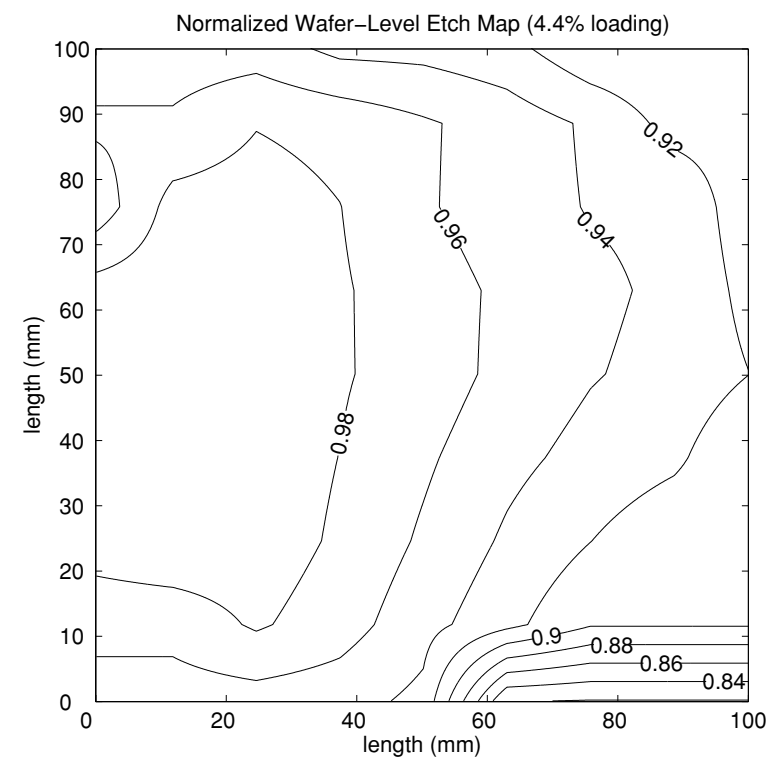

Figure 3. Normalized wafer-level etch map for $4.4 \%$ loading.

\section{MODEL APPLICATION}

While DRIE has been successful in a research environment, issues with uniformity remain a challenge for use in high volume MEMS manufacturing. An example of such a barrier can be found in the fabrication of microscale rotating power devices in silicon. These devices consist of bulk-micromachined layers bonded together, and rely heavily on DRIE. Etch nonuniformity can create variation in feature height, which may lead to imbalance in rotation and device failure [6]. The etch depth variation possible in a single device, based on WYKO measurement, is shown in Fig. 4. The individual turbopump is replicated multiple times on the wafer, resulting in a local density map as shown in Fig. 5.

The extraction shown in Fig. 5 is performed on a $100 \mu \mathrm{m}$ by $100 \mu \mathrm{m}$ grid. The white areas represent an open etch area, while the black areas represent a masked area. The local density map is convolved with the impulse response to obtain the percentage variation due to pattern density. The result is added to the normalized global etch map corresponding to the layout $(4.4 \%$ loading in the case), with rotation.

The etch variation map was compared to a wafer etched for 90 minutes with a layout based on the extraction given in Fig. 4. The chamber parameters are identical to those used to obtain the filter coefficients. The wafer was rotated $90^{\circ}$ four times to control the effects of wafer-level variation. Fig. 4 is an experimentally obtained spatial etch map for the upper left die in the layout. The etch depths range from $250 \mu \mathrm{m}$ to $268 \mu \mathrm{m}$, resulting in $6.7 \%$ across die variation. Fig. 6 shows a close-up of the same die based on pattern density model predictions, which predicts an across die variation of $7 \%$. Predictions for the turbopump mask, based on filter coefficients tuned using the characterization mask, are within $1 \%$ root-mean-square (RMS) error.

As a further test, etch rate variations are also considered on a scale internal to a single die. In order to measure intra-die variation, depth measurements were taken at a $2.2 \mathrm{~mm}$ radius from the center of each die. Taking measurements at a constant radius minimizes feature-level effects, since the feature opening will be similar along the circumference. The numbering scheme for these positions is shown in Fig. 7.

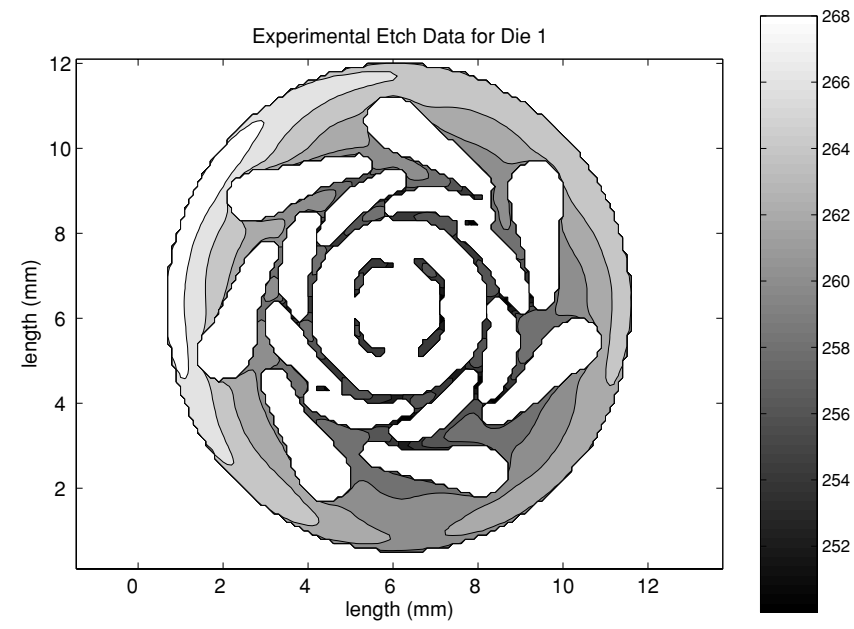

Figure 4. Measured etch depths (in $\mu m$ ) for a turbopump pattern. A $6.7 \%$ across-die variation is observed.

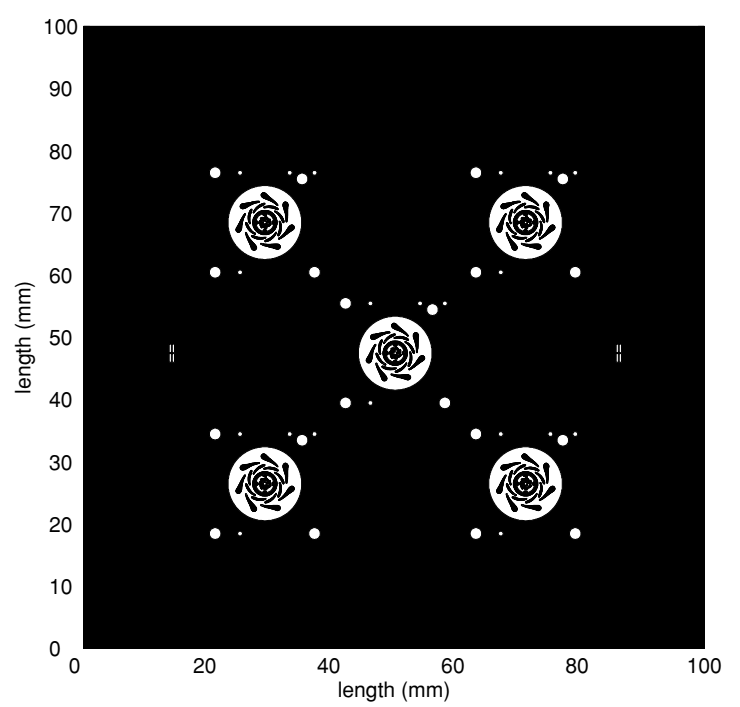

Figure 5. Image of pattern density extraction resulting from AutoCAD layout of turbopump.

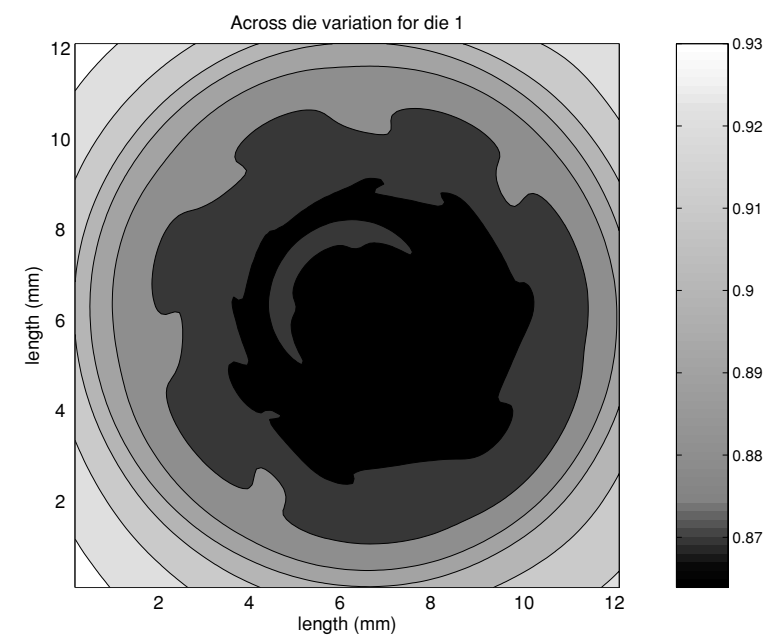

Figure 6. Spatial map of etch variation predicted by the pattern density model. The scale represents normalized etch rate. The model predicts across die variation of $7 \%$. 
13

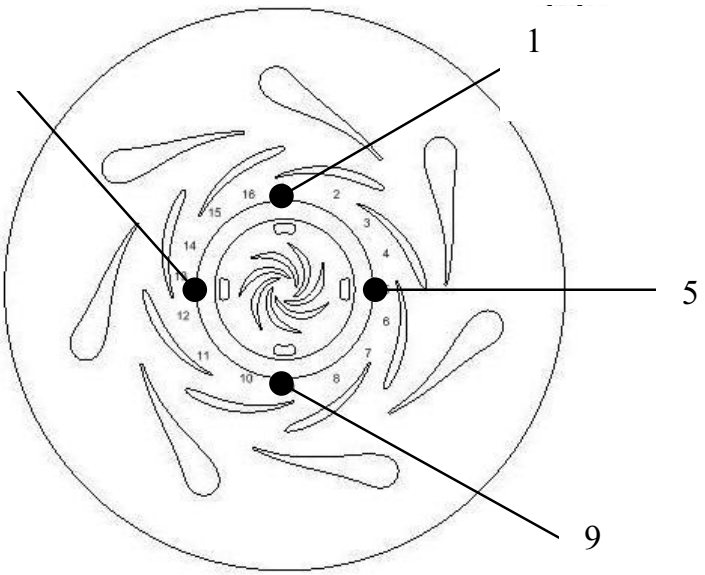

Figure 7. Experimental measurement positions for Fig. 8. The measurements were taken at a $2.2 \mathrm{~mm}$ radius from the die center, with a phase difference of $\pi / 8$ radians between them.

The pattern density model is able to capture the subtle spatial pattern of etch nonuniformity within this ring, as seen in Fig. 8 which shows predictions and experimental measurements for the upper left die. The trends in the figure can be understood qualitatively: positions nearest to the center of the layout have a higher pattern density, and experience a slower etch rate. The empirical scaling coefficient $\alpha$ is tuned to the turbopump data; without tuning, the correct spatial trends are captured but our $1 \%$ error results in offsets in the trends of Fig. 8. The filter magnitude tuning appears necessary to overcome model limitations. An $\alpha$ value of 2.34 was found to fit the data, with a normalized rootmean-square (RMS) error of $0.087 \%$ for the die.

\section{LIMITATIONS AND FUTURE WORK}

A pattern density based model has been presented for spatial mapping of layout dependent effects in DRIE. The model is able to capture the large across-die variations (6-7\%) existing in DRIE applications, such as the turbopump. The model can also capture subtle within-die effects, with additional tuning to achieve $0.1 \%$ accuracy. The pattern density model can serve as the basis for layout optimization (e.g. dummy fill generation [7]) to improve DRIE uniformity.

Several limitations and possibilities for future improvements can be noted. First, alternative functional forms for the pattern density filter function (e.g. $1 / r^{b}$ ) may be considered, or may apply in different etch circumstances. Second, feature-level or aspectratio dependent effects have not been included. Work is needed to understand how pattern density effects evolve over time as feature aspect ratios change. Third, the wafer-level model can be improved to predict wafer uniformity across varying global etch loadings by way of an improved physical model. Finally, alternative ways of combining these three components (e.g. in a multiplicative rather than additive manner) can be considered to account for wafer-, die-, and feature-level interactions.

\section{ACKNOWLEDGEMENTS}

This work was supported in part by the Cambridge-MIT Institute. The turbopump layout is courtesy of the MIT Microengine Project. We also thank Praesagus, Inc. for assistance with layout file extractions.

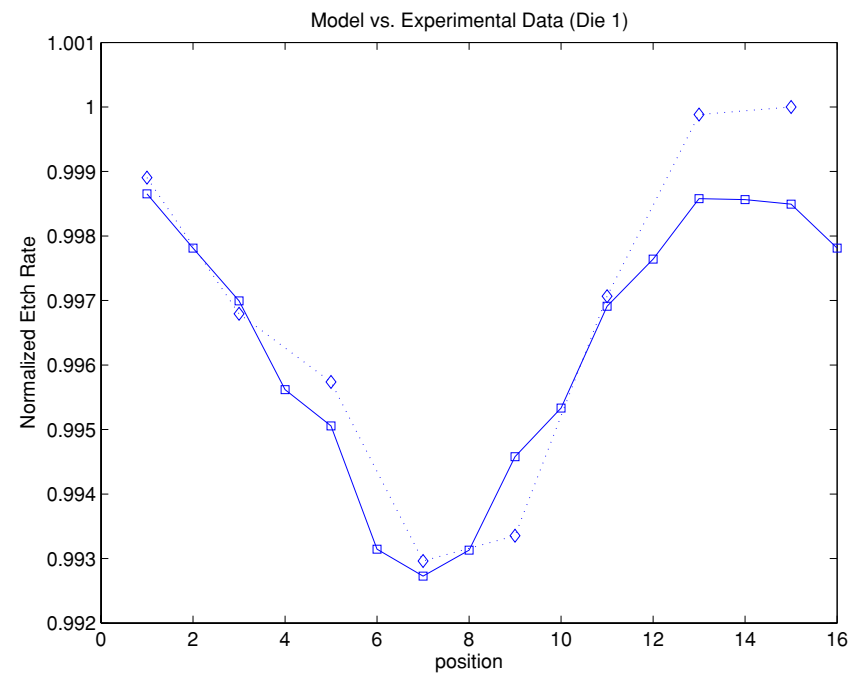

Figure 8. Comparison between model (squares) and experimental data (diamonds) for the upper left die, normalized to the fastest etching location in the die.

\section{REFERENCES}

1. H. Sun, T. Hill, M. A. Schmidt, and D. Boning, "Characterization and Modeling of Wafer and Die Level Uniformity in Deep Reactive Ion Etching (DRIE)," 2003 MRS Fall Meeting, Boston, MA, In press, Dec. 2003.

2. J. Yeom, Y. Wu, M. A. Shannon, "Critical Aspect Ratio Dependence in Deep Reactive Ion Etching of Silicon," $12^{\text {th }}$ Int. Conf. on Solid State Sensors, Actuators, and Microsystems, Boston, MA, June 2003.

3. A. Ayon, R. Bayt, C.C. Lin, R.A. Braff, H. Sawin, and M. A. Schmidt, "Characterization of a Time Multiplexed Inductively Coupled Plasma Etcher," J. Electrochem. Soc., vol. 146, no. 1, pp. 339-349, 1999.

4. D. O. Ouma, D. S. Boning, J. E. Chung, W. G. Easter, V. Saxena, S. Misra, and A. Crevasse, "Characterization and Modeling of Oxide Chemical Mechanical Polishing Using Planarization Length and Pattern Density Concepts," IEEE Trans. on. Semicond. Manuf., vol. 15, no. 2, pp. 232-244, May 2002.

5. J. W. Coburn and H. F. Winters, "Conductance considerations in the reactive ion etching of high aspect ratio features," Appl. Phys. Lett., vol. 55, no. 26, pp. 2730-2732, Dec. 1989.

6. N. Miki, C. J. Teo, L. Ho, and X. Zhang, "Precision Fabrication of High-Speed Micro-Rotors using Deep Reactive Ion Etching (DRIE)," Tech. Digest of 2002 Hilton Head SolidState Sensors and Actuators Workshop, Hilton Head Island, SC, pp. 265-268, June 2002.

7. Y. Chen, A. Kahng, G. Robins, and A. Zelikovsky, "Area Fill Synthesis for Uniform Layout Density," IEEE Trans. on CAD, vol. 21, no. 10, pp. 1132-1147, Oct. 2002.

8. C. Gormley, K. Yallup, W. Nevin, J. Bhardwaj, H. Ashraf, P. Hugget, and S. Blackstone, "State of the Art Deep Silicon Anisotropic Etching on SOI Bonded Substrates for Dielectric Isolation and MEMS Applications," Fall Meeting of the Electrochem. Society, Hawaii, USA, pp. 350-661, Oct. 1999.

9. R. A. Gottscho and C. W. Jurgensen, "Microscopic Uniformity in Plasma Etching," J. Vac. Sci. Technology B, vol. 10, no. 5, Sept./Oct. 1992. 